\title{
New neutron cross section measurements from ORELA and new resonance parameter evaluations
}

\author{
K.H. Gubera, P.E. Koehler, D. Wiarda, J.A. Harvey, T.E. Valentine, R.O. Sayer, L.L. Leal, N.M. Larson, T.S. Bigelow, \\ C. Ausmus, D.R. Brashear, R.B. Overton, J.A. White, and V.M. Cauley
}

Oak Ridge National Laboratory, P.O. Box 2008, Oak Ridge, TN 37831-6356, USA

\begin{abstract}
A series of new measurements has been undertaken in response to deficiencies identified in nuclear data libraries of crucial importance to the Nuclear Criticality Safety Program. New data and evaluations, including covariances, are required for several materials found in mixtures with uranium. For this purpose we performed neutron capture and total cross-section measurements on natural potassium, ${ }^{41} \mathrm{~K}$, and manganese.
\end{abstract}

\section{Introduction}

Many neutron cross-section evaluations in libraries such as US Evaluated Nuclear Data File (ENDF/B-VI) or Japanese Evaluated Nuclear Data File (JENDL-3.2) show deficiencies or do not cover energy ranges important for modern criticality safety calculations. Inadequacies may occur in the resolved and unresolved resonance regions. Thus, some of the evaluated data may be insufficient for criticality calculations where effects such as self-shielding, multiple scattering, or Doppler broadening are important. Moreover, for nuclides with small neutron capture cross sections, the evaluations report erroneously large values due to underestimated neutron sensitivity of the experimental set up. Although their neutron capture cross sections are small, these nuclides are sometimes absorbers in criticality calculations, and accurate cross-section data are essential. Such concerns about existing nuclear data have been the prime motivator for new cross-section measurements for the Nuclear Criticality Safety Program (NCSP) of the US Department of Energy. Criticality safety calculations involving nuclear material outside reactors have significant contributions in the epithermal energy region ( $\mathrm{keV}$ region). Many neutron cross sections are not known with sufficient accuracy. For example, due to their uncertainties in previous measurements, new neutron capture and total cross section measurements are needed for ${ }^{39,41} \mathrm{~K}$ and ${ }^{55} \mathrm{Mn}$.

\section{Measurements}

Neutron capture and total cross-section measurements for ${ }^{39,41} \mathrm{~K}$ and ${ }^{55} \mathrm{Mn}$ were performed at the Oak Ridge Electron Linear Accelerator (ORELA). ORELA was operated at a repetition rate of $525 \mathrm{~Hz}$, a burst width of $8 \mathrm{~ns}$, and a power of 5 to $6 \mathrm{~kW}$. The combination of short pulse width and long flight path resulted in a neutron energy resolution superior to any previously reported measurements for these nuclides.

Neutron capture cross sections for ${ }^{39,41} \mathrm{~K}$ and $\mathrm{Mn}$ were measured on flight path 7 at a distance of $40 \mathrm{~m}$ from the

${ }^{a}$ Presenting author, e-mail: guberkh@ornl.gov neutron-producing target using a pair of deuterated benzene $\left(\mathrm{C}_{6} \mathrm{D}_{6}\right)$ detectors by employing the pulse-height-weighting method. This apparatus [1] has been improved in several ways, compared to that used in previous measurements at ORELA for these nuclides. Firstly, the original $\mathrm{C}_{6} \mathrm{~F}_{6}$ scintillator was replaced by $\mathrm{C}_{6} \mathrm{D}_{6}$, which is much less sensitive to backgrounds from scattered neutrons. Secondly, much of structural material surrounding the sample and detectors was removed to reduce the neutron sensitivity even further. For example, the massive Al-sample changer and beam pipe were replaced by a thin carbon fiber tube. Additionally, the massive detector housings were replaced with reduced-mass detector mounts. Thirdly, the appropriate detector weighting function for each experiment is now calculated using the more sophisticated computer codes EGS4 and MCNP. All structural materials within $30 \mathrm{~cm}$ of the detectors, including the sample, are incorporated into these calculations. More details about these improvements can be found in the papers by Koehler et al. [1,2].

The neutron flux was measured using a $0.5-\mathrm{mm}$-thick ${ }^{6} \mathrm{Li}$ glass scintillator at a distance of $39.695 \mathrm{~m}$ from the neutron target. The final normalization of the capture efficiency was carried out in a separate measurement using the "saturated resonance" technique by means of the $4.9-\mathrm{eV}$ resonance from a gold sample [3].

The neutron capture cross section of ${ }^{55} \mathrm{Mn}$ was measured using a solid metallic ${ }^{55} \mathrm{Mn}$ sample with dimensions of $2.54 \times 5.08 \times 0.317 \mathrm{~cm}$. The experimental data are shown in figure 1 and compared to the cross section calculated using SAMMY [4] with ENDF/B-VI resonance parameters. This calculation included experimental effects such as selfshielding and multiple scattering. There are substantial differences between ENDF and the data, including missing resonances in the evaluation.

For ${ }^{39} \mathrm{~K}$ we used a natural potassium carbonate sample with a thickness of $0.0088 \mathrm{at} / \mathrm{b}$. The ${ }^{41} \mathrm{~K}$ neutron capture experiments using ${ }^{41} \mathrm{KCl}$ were performed with a sample enriched to $99.17 \%$ in ${ }^{41} \mathrm{~K}$ and a thickness of $0.005617 \mathrm{at} / \mathrm{b}$. Even though the sample contained chlorine, it will be possible to extract reliable resonance parameters for ${ }^{41} \mathrm{~K}$ using SAMMY by including the most recent chlorine resonance parameters [5] in the analysis. The neutron capture cross sections obtained from this experiment show that there are discrepancies compared 


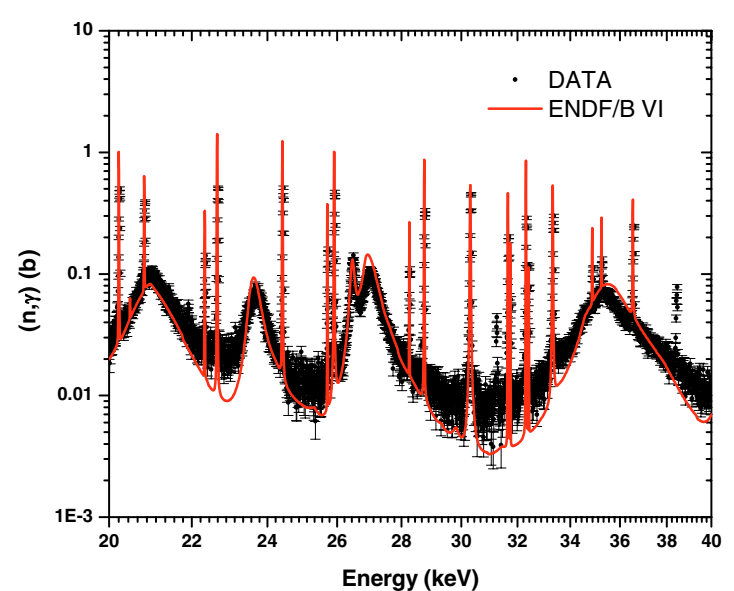

Fig. 1. New ORELA neutron capture data for Mn compared to the calculated cross section using ENDF/B VI parameters.

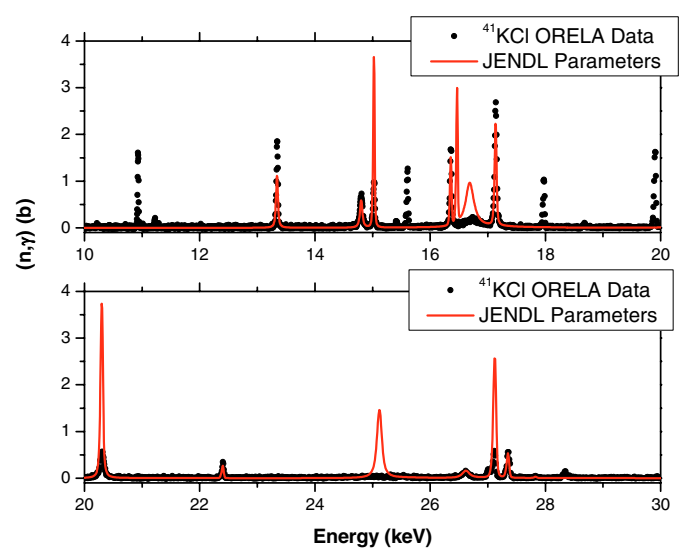

Fig. 2. New ORELA neutron capture data for ${ }^{41} \mathrm{KCl}$ compared to the calculated cross section using the JENDL 3.2 parameters.

to the cross section calculated using the resonance parameters from the JENDL 3.2 library. Figure 2 shows a small part of the data from this measurement, illustrating that many resonances for ${ }^{41} \mathrm{~K}$ are missing from the evaluation and that several resonances in the evaluation have been overestimated. The calculated cross section included experimental effects such as self-shielding, multiple scattering, and Doppler and resolution broadening.

Usually neutron capture experiments are performed with a fairly thick sample; therefore, the corrections for selfshielding and multiple scattering can be sizeable. To apply the most accurate corrections for these experimental effects, we made corresponding total cross-section measurements. These data also can be useful in making isotopic assignments as well as for observing some resonances not visible in the (n, $\gamma$ ) data. For the total cross-section measurements on $\mathrm{Mn}$, we used a metallic powder sample having a thickness of $0.119 \mathrm{at} / \mathrm{b}$. It was mounted in a computer-controlled sample changer positioned at about $10 \mathrm{~m}$ from the neutron target in the beam of ORELA. The natural potassium transmission measurement was carried out using two metallic samples $(0.013367$ and 0.10517 atom/b). For ${ }^{41} \mathrm{~K}$ we used the same ${ }^{41} \mathrm{KCl}$ material

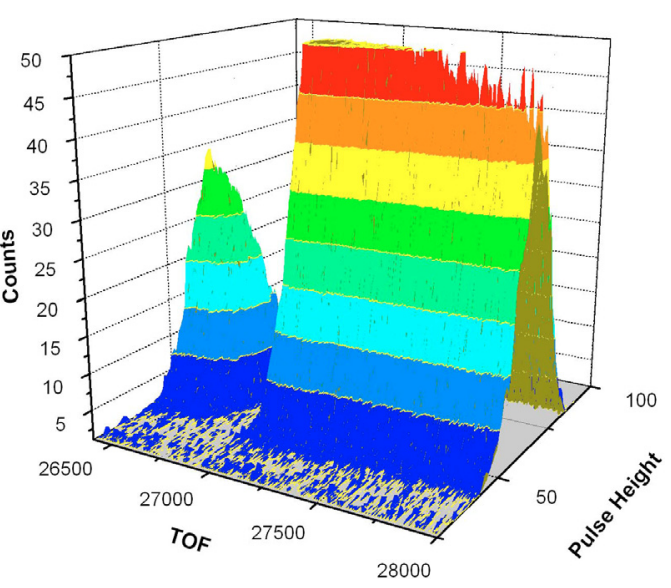

Fig. 3. Transmission data for ${ }^{41} \mathrm{KCl}$ acquired with the new data acquisition system, which records pulse-height vs TOF.

as in the capture experiments after it was reshaped to yield a thickness of $0.0155 \mathrm{at} / \mathrm{b}$. A presample collimator limited the beam size to $2.54 \mathrm{~cm}$ at the sample position and allowed only neutrons from the water-cooled part of the neutron source to be seen by the detector. As a neutron detector we used an 11.1$\mathrm{cm}$-diameter, $1.25-\mathrm{cm}$-thick ${ }^{6} \mathrm{Li}$-glass scintillator positioned in the beam at the $80-\mathrm{m}$ flight station at a distance of $79.815 \mathrm{~m}$ from the neutron source.

The total cross section was measured using a modernized data acquisition apparatus. Two channels of an Acqiris digitizer were used to digitize signals from the ${ }^{6} \mathrm{Li}$-glass detector and determine both time-of-flight (TOF) and pulse height for each event. The data were written to disk in event mode as well as displayed online in histograms. By using the digitizer many of the previous data acquisition electronics were removed; therefore the new system with fewer components should be much more reliable. A small portion of the two-dimensional data, TOF vs pulse-height, is shown in figure 3 for the ${ }^{41} \mathrm{KCl}$ transmission measurements.

\section{Evaluation}

Resonance analyses were carried out with the computer code SAMMY, which utilizes Bayes' method, a generalized leastsquares technique. Doppler and resolution broadening, multiple scattering corrections, and other effects were taken into account. Our evaluation for $\mathrm{K}$ includes our new data mentioned above as well as previous total cross-section data for enriched $\mathrm{KCl}$ samples [6]. A measurement from the Research Center at Karlsruhe (FZK) was included to extend the resolved resonance energy range up to $1.0 \mathrm{MeV}$ [7].

In figure 4 a preliminary SAMMY fit to neutron capture data using the $\mathrm{K}_{2} \mathrm{CO}_{3}$ sample is shown. Because multiple isotopes can be included in SAMMY, it is possible to analyze mixtures of isotopes and even chemicals as it is the case for the $\mathrm{KCl}$ samples. To fit those samples, the most recent chlorine resonance parameters were included in the parameter file and kept fixed. This feature in SAMMY makes it possible to extract the resonance parameters of the isotope of interest. Preliminary fits to the transmission data for ${ }^{39,41} \mathrm{KCl}$ samples 


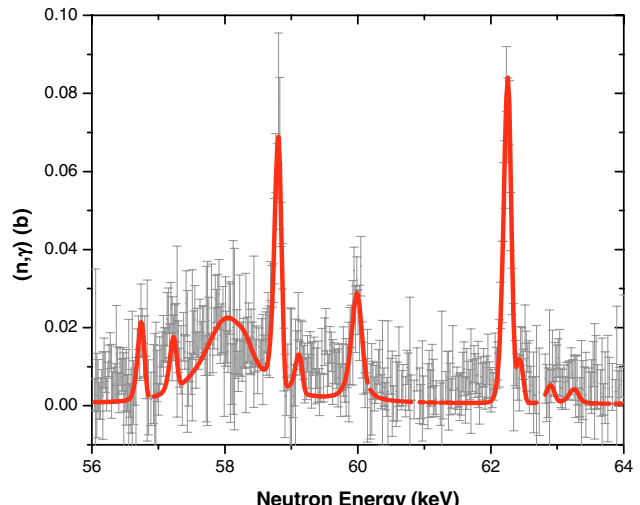

Fig. 4. Preliminary SAMMY fit to the neutron capture data for $\mathrm{K}_{2} \mathrm{CO}_{3}$ taken at ORELA.

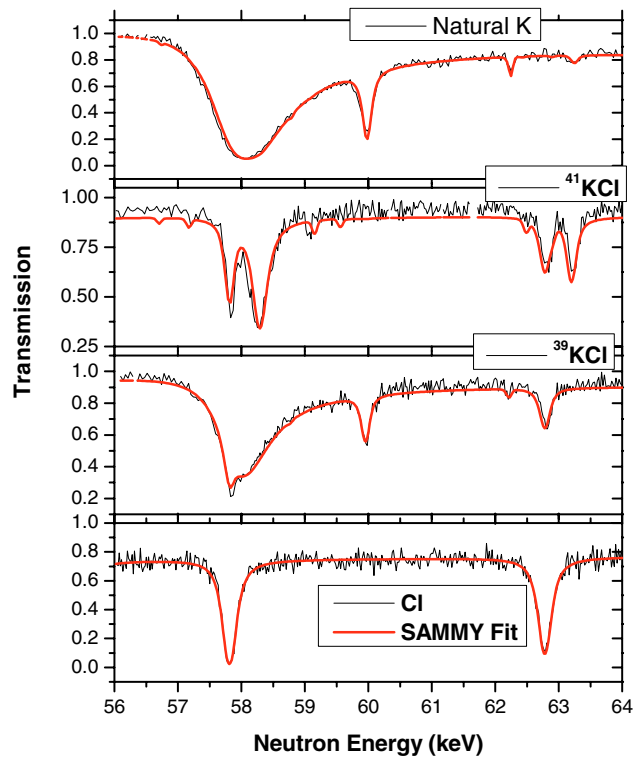

Fig. 5. Preliminary SAMMY fit to the transmission data for natural $\mathrm{K},{ }^{41} \mathrm{KCl},{ }^{39} \mathrm{KCl}$, and $\mathrm{Cl}$.

are shown together with the $\mathrm{Cl}$ transmission data in figure 5 . We expect that our new data and resonance analysis will result in improved resonance parameters for $\mathrm{K}$ and hence improved criticality safety calculations for systems containing $\mathrm{K}$.

\section{Conclusion}

To support the Nuclear Criticality Safety Program, we performed new neutron total and capture measurements over broad energy ranges for $\mathrm{K}$ and $\mathrm{Mn}$. These data will complete a series of measurements made at ORELA to be used in an evaluation of the neutron capture and total cross sections of ${ }^{39,41} \mathrm{~K}$ and $\mathrm{Mn}$. With these new data it will be possible to obtain improved resonance parameter sets for potassium and manganese in the resolved and unresolved resonance range using the multilevel R-matrix code SAMMY. In all cases we were able to extend the resolved resonance region to much higher energies compared to the existing evaluations. These new evaluations should lead to much more reliable nuclear criticality calculations.

ORNL is managed by UT-Battelle, LLC, for the US Department of Energy (DOE) under Contract No. DE-AC05-00OR22725. The work that is presented in this paper was sponsored by the DOE Nuclear Criticality Safety Program.

\section{References}

1. P.E. Koehler, R.R. Spencer, R.R. Winters, K.H. Guber, J.A. Harvey, N.W. Hill, M.S. Smith, Phys. Rev. C 54, 1463 (1996).

2. P.E. Koehler, R.R. Winters, K.H. Guber, T. Rauscher, J.A. Harvey, S. Raman, R.R. Spencer, J.C. Blackmon, D.C. Larson, D.W. Bardanyan, T.A. Lewis, Phys. Rev. C 62, 055803-1 (2000).

3. R.L. Macklin, J. Halperin, R.R. Winters, Nucl. Instrum. Meth. 164, 213 (1979).

4. N.M. Larson, ORNL/TM-9179/R7, Oak Ridge National Laboratory, 2006.

5. R.O. Sayer, K.H. Guber, L.C. Leal, N.M. Larson, Phys. Rev. C 73, 044603 (2006).

6. W.M. Good, J.A. Harvey, N.W. Hill, ORNL-4937, UC-34Physics, Oak Ridge National Laboratory, 1974, and J.A. Harvey, 2005 (private communication).

7. S. Cierjacks, Kernforschungs Zentrum Karlsruhe, Report KFK1000 (Supp. 2), 1969. 